\title{
Generalized Derivations on (Semi-)Prime Rings and Noncommutative Banach Algebras
}

\author{
Feng Wei $(*)$ - Zhankui Xiao (*)
}

ABSTRACT - We first give several polynomial identities of semiprime rings which make the additive mappings appearing in the identities to be generalized derivations. Then we study some pairs of generalized Jordan derivations with power values on prime rings. Let $m, n$ be fixed positive integers, $\mathcal{R}$ be a noncommutative $2(m+n)$ !-torsion free prime ring with the center $\mathcal{Z}$ and $\mu, v$ be a pair of generalized Jordan derivations on $\mathcal{R}$. If $\mu\left(x^{m}\right) x^{n}+x^{n} v\left(x^{m}\right) \in \mathcal{Z}$ for all $x \in \mathcal{R}$, then $\mu$ and $v$ are left (or right) multipliers. In particular, if $\mu, v$ are a pair of derivations on $\mathcal{R}$ satisfying the same assumption, then $\mu=v=0$. Then applying these purely algebraic result we obtain several range inclusion results of pair of derivations on Banach algebras.

\section{Introduction.}

Let $\mathcal{R}$ be an associative ring. An additive mapping $\mu: \mathcal{R} \rightarrow \mathcal{R}$ is called a generalized derivation of $\mathcal{R}$ if there exists a derivation $d$ of $\mathcal{R}$ such that $\mu(x y)=\mu(x) y+x d(y)$ for all $x, y \in \mathcal{R}$, where $d$ is called the associated derivation of $\mu$. If $\mu$ is a generalized derivation of $\mathcal{R}$ with the associated derivation $d$, then $\mu$ satisfies the following relations

$$
\mu\left(x^{n}\right)=\mu(x) x^{n-1}+\sum_{j=1}^{n-1} x^{n-j} d(x) x^{j-1}
$$

and

$$
\mu\left(x^{n}\right)=\mu\left(x^{n-m}\right) x^{m}+x^{n-m} d\left(x^{m}\right)
$$

(*) Indirizzo degli A.: Department of Applied Mathematics, Beijing Institute of Technology, Beijing, 100081, P. R. China.

E-mail: daoshuo@bit.edu.cn zhkxiao@bit.edu.cn

2000 Mathematics Subject Classification. 16W25, 16N60. 
for all $x \in \mathcal{R}$, where $m, n$ are positive integers with $1 \leq m \leq n-1$. It seems natural to ask under what additional assumptions the converse is true. More precisely, under what additional assumptions an additive mapping $\mu$, which maps a ring $\mathcal{R}$ into itself, satisfying one of the relations (0.1) and (0.2) is a generalized derivation. Bridges and Bergen [4], Vukmann and Kosi-ulbl [25] have considered the similar problems for the case of derivations on rings. In this paper we will give affirmative answers to just mentioned questions under mild conditions.

Inspired by the relations (0.1) and (0.2) we consider much more common generalized forms of skew centralizing mappings involved pair of (generalized-)Jordan derivations with power values on a prime ring $\mathcal{R}$. Precisely speaking, the generalized forms are

(a) $\mu\left(x^{m}\right) x^{n}+x^{n} v\left(x^{m}\right) \in \mathcal{Z}$;

(b) $d\left(x^{m}\right) x^{n}+x^{n} g\left(x^{m}\right) \in \mathcal{Z}$;

(c) $x^{m} d(x) x^{n}+x^{p} g(x) x^{q} \in \mathcal{Z}$,

where $m, n, p, q$ are positive integers, $\mu, v$ are a pair of generalized Jordan derivations on $\mathcal{R}, d, g$ are a pair of Jordan derivations on $\mathcal{R}$ and $\mathcal{Z}$ is the center of $\mathcal{R}$. In fact, some similar identities with (generalized-)derivations have been studied by many people in various way, see [1], [9], [11], [14], [24], [27] and [28]. In the present paper we prove the following results. Let $\mathcal{R}$ be a noncommutative $2(m+n)$ !-torsion free prime ring . If (a) holds for all $x \in \mathcal{R}$, then $\mu$ and $v$ are left (or right) multipliers. In particular, if (b) holds for all $x \in \mathcal{R}$, then $d=g=0$. If (c) holds for all $x \in \mathcal{R}$, then $d=g=0$. All these results generalize many existed conclusions in this field.

On the other hand, many authors use pure algebraic techniques (especially, the techniques concerning (semi-)prime rings) to study the range inclusion problem of derivations on Banach algebras, which are related to the well known noncommutative Singer-Wermer conjecture. Various partial answers to this conjecture are obtained. Most results in the field of range inclusion deal with one derivation, while pair of derivations on Banach algebras was more less considered. In this paper we apply the algebraic results in the previous paragraph to discuss pairs of derivations on Banach algebras. Let $\mathcal{A}$ be a noncommutative Banach algebra with the Jacobson radical $\operatorname{rad}(\mathcal{A})$ and $d, g$ be a pair of derivations on $\mathcal{A}$. If $d\left(x^{m}\right) x^{n}+x^{n} g\left(x^{m}\right) \in \mathcal{Z}_{\mathcal{A}}$ for all $x \in \mathcal{A}$ and fixed positive integers $m$, $n$, then $d(\mathcal{A}) \subseteq \operatorname{rad}(\mathcal{A})$ and $g(\mathcal{A}) \subseteq \operatorname{rad}(\mathcal{A})$. The case of a pair of Jordan derivations on Banach algebras is our next goal. Let $\mathcal{A}$ be a noncommutative Banach algebra with the Jacobson radical $\operatorname{rad}(\mathcal{A})$ and $d, g$ be 
a pair of continuous Jordan derivations on $\mathcal{A}$. If $d\left(x^{m}\right) x^{n}+x^{n} g\left(x^{m}\right) \in$ $\in \operatorname{rad}(\mathcal{A})$ for all $x \in \mathcal{A}$ and fixed positive integers $m, n$, then $d(\mathcal{A}) \subseteq \operatorname{rad}(\mathcal{A})$ and $g(\mathcal{A}) \subseteq \operatorname{rad}(\mathcal{A})$.

\section{Some Additive Mappings are Generalized Derivations.}

Throughout this section $\mathcal{R}$ always denotes an associative ring. If $\mathcal{R}$ has the identity element $e$, we can adopt the convention that $x^{0}=e$ for all $x \in \mathcal{R} \backslash\{0\}$. Recall that a ring $\mathcal{R}$ is prime if for all $a, b \in \mathcal{R}, a \mathcal{R} b=0$ implies that either $a=0$ or $b=0$, and is semiprime in case $a \mathcal{R} a=0$ implies that $a=0$. An additive mapping $d: \mathcal{R} \rightarrow \mathcal{R}$ is said to be a derivation if $d(x y)=d(x) y+x d(y)$ for all for $x, y \in \mathcal{R}$ and is called a Jordan derivation if $d\left(x^{2}\right)=d(x) x+x d(x)$ for all $x \in \mathcal{R}$. Every derivation is a Jordan derivation, but the converse is in general not true. A classical result of Herstein [12] asserts that any Jordan derivation on a 2 -torsion free prime ring is a derivation. Cusack [6] extended Herstein's result to the case of semiprime rings and proved that every Jordan derivation on a 2 -torsion free semiprime ring is a derivation. Let $\mu$ be a generalized derivation of $\mathcal{R}$ with the associated derivation $d$. Then $\mu$ satisfies the relation (0.1). We find that under additional assumptions, the converse is also true for a class of additive mappings on semiprime rings.

THeOREm 1.1. Let $n>1$ be a fixed positive integer and $\mathcal{R}$ be a $n$ !torsion free semiprime ring with the identity element . Suppose that there exist additive mappings $\mu: \mathcal{R} \rightarrow \mathcal{R}, d: \mathcal{R} \rightarrow \mathcal{R}$ such that

$$
\mu\left(x^{n}\right)=\mu(x) x^{n-1}+\sum_{j=1}^{n-1} x^{n-j} d(x) x^{j-1}
$$

for all $x \in \mathcal{R}$. Then $\mu$ is a generalized derivation on $\mathcal{R}$ with the associated derivation $d$.

Proof. The assumption implies that

$$
\mu\left(x^{n}\right)-\mu(x) x^{n-1}-\sum_{j=1}^{n-1} x^{n-j} d(x) x^{j-1}=0
$$

for all $x \in \mathcal{R}$. By (1.1) we immediately get $d(e)=0$. Substituting $x+\lambda y$ for $x$ in (1.1) we obtain

$$
\lambda P_{1}(x, y)+\lambda^{2} P_{2}(x, y)+\cdots+\lambda^{n} P_{n}(x, y)=0,
$$


where $\lambda \in Z, x, y \in \mathcal{R}, P_{i}(x, y)$ denotes the sum of terms involving $i$ factors of $y$ in the expansion of $\mu\left((x+\lambda y)^{n}\right)-\mu(x+\lambda y)(x+\lambda y)^{n-1}-\sum_{j=1}^{n-1}(x+\lambda y)^{n-j} d(x+\lambda y)(x+\lambda y)^{j-1}=0$.

By [5, Lemma 1] it follows that

$$
\begin{aligned}
P_{1}(x, y)= & \mu\left(x^{n-1} y+x^{n-2} y x+\cdots+y x^{n-1}\right)-\mu(y) x^{n-1} \\
& -\mu(x)\left(x^{n-2} y+x^{n-3} y x+\cdots+y x^{n-2}\right) \\
& -\sum_{j=1}^{n-1}\left(x^{n-j-1} y+\cdots+y x^{n-j-1}\right) d(x) x^{j-1}-\sum_{j=1}^{n-1} x^{n-j} d(y) x^{j-1} \\
& -\sum_{j=1}^{n-1} x^{n-j} d(x)\left(x^{j-2} y+x^{j-3} y x+\cdots+y x^{j-2}\right)=0
\end{aligned}
$$

for all $x, y \in \mathcal{R}$. Taking $x=e$ into (1.2) leads to

$$
n \mu(y)-\mu(y)-(n-1) \mu(e) y-(n-1) d(y)=0
$$

for all $y \in \mathcal{R}$. Note that $\mathcal{R}$ is $n$ !-torsion free. So we have

$$
\mu(y)=\mu(e) y+d(y)
$$

for all $y \in \mathcal{R}$. Replacing $y$ by $x^{n}$ in (1.3) yields

$$
\mu\left(x^{n}\right)=\mu(e) x^{n}+d\left(x^{n}\right)
$$

for all $x \in \mathcal{R}$. This implies that

$$
\mu(e) x^{n}+d\left(x^{n}\right)=\mu(x) x^{n-1}+\sum_{j=1}^{n-1} x^{n-j} d(x) x^{j-1}
$$

for all $x \in \mathcal{R}$. Combining (1.4) with (1.3) we get

$$
d\left(x^{n}\right)=\sum_{j=1}^{n} x^{n-j} d(x) x^{j-1}
$$

for all $x \in \mathcal{R}$. Substituting $x+\lambda y$ for $x$ in (1.5) leads to

$$
\lambda P_{1}(x, y)+\lambda^{2} P_{2}(x, y)+\cdots+\lambda^{n} P_{n}(x, y)=0,
$$

where $\lambda \in Z, x, y \in \mathcal{R}, P_{i}(x, y)$ denotes the sum of terms involving $i$ factors of $y$ in the expansion of

$$
d\left((x+\lambda y)^{n}\right)-\sum_{j=1}^{n}(x+\lambda y)^{n-j} d(x+\lambda y)(x+\lambda y)^{j-1}=0 .
$$


By [5, Lemma 1] again we obtain

$$
P_{2}(e, y)=\frac{n(n-1)}{2} d\left(y^{2}\right)-\sum_{j=1}^{n}(n-j) y d(y)-\sum_{j=1}^{n}(j-1) d(y) y=0
$$

for all $y \in \mathcal{R}$. Note that $\mathcal{R}$ is $n$ !-torsion free. So

$$
d\left(y^{2}\right)=d(y) y+y d(y)
$$

for all $y \in \mathcal{R}$. This shows that $d$ is a Jordan derivation on $\mathcal{R}$ and hence is a derivation on $\mathcal{R}$. Thus the identity (1.3) shows that $\mu$ is a generalized derivation on $\mathcal{R}$ with the associated derivation $d$.

As a direct corollary of Theorem 1.1 we have

CoRollary 1.2 [25, Theorem 1]. Let $n>1$ be a fixed positive integer and $\mathcal{R}$ be a $n$ !-torsion free semiprime ring with the identity element $e$. Suppose that there exists an additive mapping $d: \mathcal{R} \rightarrow \mathcal{R}$ such that

$$
d\left(x^{n}\right)=\sum_{j=1}^{n} x^{n-j} d(x) x^{j-1}
$$

for all $x \in \mathcal{R}$. Then $d$ is a derivation on $\mathcal{R}$.

According to the definition of generalized derivation, if $\mu$ is a generalized derivation of $\mathcal{R}$ with the associated derivation $d$, then $\mu$ also satisfies another relation (0.2). Analogously, under mild conditions, the converse also holds for a class of additive mappings on semiprime rings.

THEOREM 1.3. Let $n>1$ be a fixed positive integer and $\mathcal{R}$ be a $n$ !torsion free semiprime ring with the identity element e. Suppose that there exist additive mappings $\mu: \mathcal{R} \rightarrow \mathcal{R}$ and $d: \mathcal{R} \rightarrow \mathcal{R}$ such that

$$
\mu\left(x^{n}\right)=\mu\left(x^{n-m}\right) x^{m}+x^{n-m} d\left(x^{m}\right)
$$

for all $x \in \mathcal{R}$, where $m$ is a positive integer with $1 \leq m \leq n-1$. Then $\mu$ is $a$ generalized derivation on $\mathcal{R}$ with the associated derivation $d$.

Proof. The assumption implies that

$$
\mu\left(x^{n}\right)-\mu\left(x^{n-m}\right) x^{m}-x^{n-m} d\left(x^{m}\right)=0
$$

for all $x \in \mathcal{R}$. Then it is easy to see that $d(e)=0$. Substituting $x+\lambda y$ for $x$ in (1.7) we obtain

$$
\lambda P_{1}(x, y)+\lambda^{2} P_{2}(x, y)+\cdots+\lambda^{n} P_{n}(x, y)=0
$$


where $\lambda \in Z, x, y \in \mathcal{R}, P_{i}(x, y)$ denotes the sum of terms involving $i$ factors of $y$ in the expansion of $\mu\left((x+\lambda y)^{n}\right)-\mu\left((x+\lambda y)^{n-m}\right)(x+\lambda y)^{m}-$ $-(x+\lambda y)^{n-m} d\left((x+\lambda y)^{m}\right)=0$. It follows from [5, Lemma 1] that

$$
P_{1}(x, y)=\mu\left(x^{n-1} y+x^{n-2} y x+\cdots+y x^{n-1}\right)
$$

$$
\begin{aligned}
-\mu\left(x^{n-m-1} y+\cdots+\right. & \left.y x^{n-m-1}\right) x^{m}-\mu\left(x^{n-m}\right)\left(x^{m-1} y+x^{m-2} y x+\cdots+y x^{m-1}\right) \\
& -\left(x^{n-m-1} y+\cdots+y x^{n-m-1}\right) d\left(x^{m}\right) \\
& -x^{n-m} d\left(x^{m-1} y+x^{m-2} y x+\cdots+x y x^{m-2}+y x^{m-1}\right)=0
\end{aligned}
$$

for all $x, y \in \mathcal{R}$. Taking $x=e$ into (1.8) yields

$$
n \mu(y)-(n-m) \mu(y)-m \mu(e) y-m d(y)=0
$$

for all $y \in \mathcal{R}$. Note that $\mathcal{R}$ is $n$ !-torsion free. So

$$
\mu(y)=\mu(e) y+d(y)
$$

for all $y \in \mathcal{R}$. Combining (1.9) with (1.7) we get

$$
d\left(x^{n}\right)=d\left(x^{n-m}\right) x^{m}+x^{n-m} d\left(x^{m}\right)
$$

for all $x \in \mathcal{R}$. Substituting $x+\lambda y$ for $x$ in (1.10) leads to

$$
\lambda P_{1}(x, y)+\lambda^{2} P_{2}(x, y)+\cdots+\lambda^{n} P_{n}(x, y)=0,
$$

where $\lambda \in \mathbb{Z}, x, y \in \mathcal{R}, P_{i}(x, y)$ denotes the sum of terms involving $i$ factors of $y$ in the expansion of $d\left((x+\lambda y)^{n}\right)-d\left((x+\lambda y)^{n-m}\right)(x+\lambda y)^{m}-$ $-(x+\lambda y)^{n-m} d\left((x+\lambda y)^{m}\right)=0$. By [5, Lemma 1] again we have

$$
\begin{aligned}
P_{2}(e, y)= & \frac{n(n-1)}{2} d\left(y^{2}\right)-\frac{(n-m)(n-m-1)}{2} d\left(y^{2}\right) \\
& -(n-m) m d(y) y-(n-m) m y d(y)-\frac{m(m-1)}{2} d\left(y^{2}\right)=0
\end{aligned}
$$

for all $y \in \mathcal{R}$. In view of the torsion free fact of $\mathcal{R}$, we see that

$$
d\left(y^{2}\right)=d(y) y+y d(y)
$$

for all $y \in \mathcal{R}$. This shows that $d$ is a Jordan derivation on $\mathcal{R}$ and hence is a derivation. Thus the identity (1.9) proves that $\mu$ is a generalized derivation on $\mathcal{R}$ with the associated derivation $d$.

As an immediate consequence of Theorem 1.3 we have

CoROLlary 1.4. Let $n>1$ be a fixed positive integer and $\mathcal{R}$ be $a n$ !torsion free semiprime ring with the identity element e. Suppose that there 
exists an additive mapping $d: \mathcal{R} \rightarrow \mathcal{R}$ such that

$$
d\left(x^{n}\right)=d\left(x^{n-m}\right) x^{m}+x^{n-m} d\left(x^{m}\right)
$$

for all $x \in \mathcal{R}$, where $m$ is a positive integer with $1 \leq m \leq n-1$. Then $d$ is $a$ derivation on $\mathcal{R}$.

An additive mapping $\mu: \mathcal{R} \rightarrow \mathcal{R}$ is called a generalized Jordan derivation of $\mathcal{R}$ if there exists a Jordan derivation $d$ of $\mathcal{R}$ such that $\mu\left(x^{2}\right)=\mu(x) x+x d(x)$ for all $x \in \mathcal{R}$, where $d$ is called the associated Jordan derivation of $\mu$. Obviously, any generalized derivation is a generalized Jordan derivation. But, the converse statement is not necessarily true. It has been proved that any generalized Jordan derivation on a 2-torsion free semiprime ring is a generalized derivation [27]. Thus, if $\mu$ is a generalized Jordan derivation on a 2-torsion free semiprime ring $\mathcal{R}$, then $\mu(x y x)=$ $=\mu(x) y x+x d(y x)$ for all $x, y \in \mathcal{R}$. Under mild assumptions, the converse also holds for a class of additive mappings on a 2-torsion free semiprime ring. But, the condition that $\mathcal{R}$ contains the identity element $e$ is not necessary here, which is different form the additional assumption of Theorem 1.1 and that of Theorem 1.3.

THEOREM 1.5. Let $\mathcal{R}$ be a 2-torsion free semiprime ring, $\mu: \mathcal{R} \rightarrow \mathcal{R}$ be an additive mapping and $d$ be a Jordan derivation of $\mathcal{R}$. If $\mu(x y x)=$ $=\mu(x) y x+x d(y x)$ for all $x, y \in \mathcal{R}$, then $\mu$ is a generalized derivation with the associated derivation $d$.

Proof. The linearization of the assumption

$$
\mu(x y x)=\mu(x) y x+x d(y x)
$$

gives

$$
\mu(x y z+z y x)=\mu(x) y z+x d(y z)+\mu(z) y x+z d(y x)
$$

for all $x, y, z \in \mathcal{R}$. Taking $z=x^{2}$ into (1.13) we obtain

$$
\mu\left(x y x^{2}+x^{2} y x\right)=\mu(x) y x^{2}+x d\left(y x^{2}\right)+\mu\left(x^{2}\right) y x+x^{2} d(y x)
$$

for all $x, y \in \mathcal{R}$. Substituting $x y+y x$ for $y$ in (1.12) yields

$$
\mu\left(x^{2} y x+x y x^{2}\right)=\mu(x) x y x+\mu(x) y x^{2}+x d(x y x)+x d\left(y x^{2}\right)
$$

for all $x, y \in \mathcal{R}$. From (1.14) and (1.15) we have

$$
A(x) y x=0
$$


for all $x, y \in \mathcal{R}$, where $A(x)$ denotes $\mu\left(x^{2}\right)-\mu(x) x-x d(x)$. It is sufficient to prove that $A(x)=0$ for all $x \in \mathcal{R}$. In (1.16), we replace $x y A(x)$ for $y$ and then get $A(x) x y A(x) x=0$ for all $x, y \in \mathcal{R}$. By the semiprimeness of $\mathcal{R}$ it follows that

$$
A(x) x=0
$$

for all $x \in \mathcal{R}$. Left multiplication of (1.16) by $x$ and right multiplication of (1.16) by $A(x)$ lead to $x A(x) y x A(x)=0$ for all $x, y \in \mathcal{R}$. In view of the semiprimeness of $\mathcal{R}$, we get

$$
x A(x)=0
$$

for all $x \in \mathcal{R}$. The linearization of (1.17) leads to

$$
A(x) y+B(x, y) x+A(y) x+B(x, y) y=0
$$

for all $x, y \in \mathcal{R}$, where $B(x, y)$ denotes $\mu(x y+y x)-\mu(x) y-\mu(y) x-$ $-x d(y)-y d(x)$. Replacing $-x$ for $x$ in (1.18), we have

$$
A(x) y+B(x, y) x-A(y) x-B(x, y) y=0
$$

for all $x, y \in \mathcal{R}$. Combining (1.19) with (1.18), we obtain

$$
A(x) y+B(x, y) x=0
$$

for all $x, y \in \mathcal{R}$. Right multiplication of the above relation by $A(x)$ leads to $A(x) y A(x)=0$ for all $x, y \in \mathcal{R}$. In view of the semiprimeness of $\mathcal{R}$, we get $A(x)=0$ for all $x \in \mathcal{R}$. This proves that $\mu\left(x^{2}\right)=\mu(x) x+x d(x)$ for all $x \in \mathcal{R}$ and that $\mu$ is a generalized Jordan derivation of $\mathcal{R}$ with the associated Jordan derivation $d$. By [27, Theorem 2.6] it follows that $\mu$ is a generalized derivation with the associated derivation $d$.

\section{Pair of Generalized Derivations on (Semi-)Prime Rings.}

Let $\mathcal{R}$ be a ring with the center $\mathcal{Z}$. A mapping $f: \mathcal{R} \longrightarrow \mathcal{R}$ is said to be skew centralizing $f(x) x+x f(x) \in \mathcal{Z}$ for all $x \in \mathcal{R}$. The study of skew centralizing mappings was motivated by a well-known theorem of Posner which says that the existence of a nonzero centralizing derivation on a prime ring $\mathcal{R}$ implies that $\mathcal{R}$ is commutative [18]. This theorem has been generalized by many researchers in different ways. One interesting branch of all related works is to consider a common generalization of skew centralizing mappings which involves pair of generalized Jordan derivations on prime rings. In this section, one much more common generalized form of skew centralizing mapping such as $\mu\left(x^{m}\right) x^{n}+x^{n} v\left(x^{m}\right) \in \mathcal{Z}$ will be stu- 
died, where $m, n$ are fixed positive integers, $\mu$ and $v$ are a pair of generalized Jordan derivations on prime ring $\mathcal{R}$.

For the proof of our main result of this section, we need some basic facts. Through out this section $\mathcal{R}$ always denotes a (semi-)prime ring and $\mathcal{U}$ is the left Utumi quotient ring of $\mathcal{R}$. $\mathcal{U}$ can be characterized as a ring satisfying the following properties:

(a) $\mathcal{R}$ is a subring of $\mathcal{U}$.

(b) For each $q \in \mathcal{U}$, there exists a dense left ideal $I_{q}$ of $\mathcal{R}$ such that $I_{q} q \subseteq \mathcal{R}$.

(c) If $q \in \mathcal{U}$ and $I q=0$ for some ideal $I$ of $\mathcal{R}$, then $q=0$.

(d) If $\phi: I \rightarrow \mathcal{R}$ is a left $\mathcal{R}$-module homomorphism from a dense left ideal $I$ of $\mathcal{R}$ into $\mathcal{R}$, then there exists an element $q \in \mathcal{U}$ such that $\phi(i)=i q$ for all $i \in I$.

Up to isomorphisms, $\mathcal{U}$ is uniquely determined by the above four properties. The center of $\mathcal{U}$ is called the extended centroid of $\mathcal{R}$ and denoted by $\mathcal{C}$. It is well known that $\mathcal{C}$ is a Von Neumann regular ring. It turns out that $\mathcal{C}$ is a field if and only if $\mathcal{R}$ is a prime ring. The set of all idempotents of $\mathcal{C}$ is denoted by $\mathcal{E}$. The element of $\mathcal{E}$ are called central idempotents.

Another related object we have to mention is the generalized differential identities on (semi-)prime rings. A generalized differential polynomial over $\mathcal{U}$ means a generalized polynomial with coefficients in $\mathcal{U}$ and with noncommutative variables involving generalized derivations. A generalized differential identity for some subset of $\mathcal{U}$ is a generalized differential polynomial satisfied by the given subset. Obviously, the definition of a generalized differential polynomial(or identity) is a common generalization of the definition of a differential polynomial(or identity). Before we state the main result of this section, several useful lemmas are given.

LEMMA 2.1. Let $n$ be a fixed positive integer, $\mathcal{R}$ be a $n$ !-torsion free ring with center $\mathcal{Z}$. Suppose $y_{1}, y_{2}, \cdots, y_{n} \in \mathcal{R}$ satisfy $\lambda y_{1}+\lambda^{2} y_{2}+\cdots+\lambda^{n} y_{n} \in \mathcal{Z}$ for $\lambda=1,2, \cdots, n$. Then $y_{i} \in \mathcal{Z}$ for all $i$.

The Lemma 1 of [5] has been used many times in section 1 and Lemma 2.1 is actually one generalization of this result. The proof of Lemma 2.1 is completely analogous to the proof of Lemma 1 of [5].

LEMMa 2.2 [26, Theorem 2]. Let $\mathcal{R}$ be a semiprime ring and I be a dense $i d e a l$ of $\mathcal{R}$. Then any generalized derivation $\mu$ on I can be uniquely extended to a generalized derivation of $\mathcal{U}$. Furthermore, the extended gen- 
eralized derivation $\mu$ has the form $\mu(x)=a x+d(x)$ for all $x \in \mathcal{U}$, where $a \in \mathcal{U}$ and $d$ is a derivation of $\mathcal{U}$.

Now we are ready to give the first main result of this paper.

THEOREM 2.3. Let $m, n$ be fixed positive integers, $\mathcal{R}$ be a noncommutative $2(m+n)$ !-torsion free prime ring and $\mu, v$ be a pair of generalized Jordan derivations on $\mathcal{R}$. If $\mu\left(x^{m}\right) x^{n}+x^{n} v\left(x^{m}\right) \in \mathcal{Z}$ for all $x \in \mathcal{R}$, then $\mu$ and $v$ are right (or left) multipliers.

Proof. Since $\mu$ and $v$ are generalized Jordan derivations on $\mathcal{R}, \mu$ and $v$ are generalized derivations on $\mathcal{R}$ by [27, Theorem 2.6]. It is well known that $\mathcal{R}$ and $\mathcal{U}$ satisfy the same differential identities [16, Theorem 2] and hence also satisfy the same generalized differential identities by Lemma 2.2. This implies that

$$
\mu\left(x^{m}\right) x^{n}+x^{n} v\left(x^{m}\right) \in \mathcal{C}
$$

for all $x \in \mathcal{U}$. Note that $\mathcal{U}$ has the identity element $e$. It is easy to see that

$$
\mu(e)+v(e) \in \mathcal{C} .
$$

Substituting $x+\lambda y$ for $x$ in (2.1) and applying Lemma 2.1, we get

$$
\begin{aligned}
& \text { (2.3) } P_{1}(x, y)=\mu\left(x^{m-1} y+x^{m-2} y x+\cdots+y x^{m-1}\right) x^{n} \\
& +\mu\left(x^{m}\right)\left(x^{n-1} y+x^{n-2} y x+\cdots+y x^{n-1}\right)+\left(x^{n-1} y+x^{n-2} y x+\cdots+y x^{n-1}\right) v\left(x^{m}\right) \\
& +x^{n} v\left(x^{m-1} y+x^{m-2} y x+\cdots+y x^{m-1}\right) \in \mathcal{C} .
\end{aligned}
$$

for all $x, y \in \mathcal{U}$, where $\lambda \in \mathbb{Z}$ and $P_{i}(x, y)$ denotes the sum of terms involving $i$ factors of $y$ in the expansion of $\mu\left((x+\lambda y)^{m}\right)(x+\lambda y)^{n}+$ $+(x+\lambda y)^{n} v\left((x+\lambda y)^{m}\right) \in \mathcal{C}$. Taking $x=e$ into (2.3) leads to

$$
m \mu(y)+n \mu(e) y+n y v(e)+m v(y) \in \mathcal{C} .
$$

for all $y \in \mathcal{U}$. It follows from (2.4) and Lemma 2.2 that

$$
(m+n) \mu(e) y+n y v(e)+m v(e) y+m d(y)+m g(y) \in \mathcal{C} .
$$

for all $y \in \mathcal{U}$, where $d$ and $g$ are the associated derivations of $\mu$ and $v$, respectively. Thus

$$
n[\mu(e) y+y v(e), y]+m[d(y)+g(y), y]=0
$$

for all $y \in \mathcal{U}$. Rewrite (2.6) as follows

$$
([n \mu(e), y]+m(d(y)+g(y))) y-y([y, n v(e)]+m(d(y)+g(y)))=0
$$


for all $y \in \mathcal{U}$. By [3, Theorem 4.1] we have

$$
\begin{aligned}
& n[\mu(e), y]+m(d(y)+g(y))=0 \\
& n[y, v(e)]+m(d(y)+g(y))=0
\end{aligned}
$$

for all $y \in \mathcal{U}$. Combining (2.8) with (2.5), we obtain

$$
m(\mu(e)+v(e)) y+n y(\mu(e)+v(e)) \in \mathcal{C} .
$$

for all $y \in \mathcal{U}$. Note that $\mu(e)+v(e) \in \mathcal{C}$ and $\mathcal{R}$ is $2(m+n)$ !-torsion free. So

$$
\mu(e)=-v(e) \text {. }
$$

By Lemma 2.2, the relation (2.1) can be set

$$
\mu(e) x^{m+n}+x^{n} v(e) x^{m}+d\left(x^{m}\right) x^{n}+x^{n} g\left(x^{m}\right) \in \mathcal{C} .
$$

Replacing $y$ by $x^{n}$ in (2.8) and then right multiplication by $x^{m}$ gives

$$
n \mu(e) x^{n+m}-n x^{n} \mu(e) x^{m}=-m g\left(x^{n}\right) x^{m}-m d\left(x^{n}\right) x^{m}
$$

for all $x \in \mathcal{U}$. According to the above relation and (2.12), we have

$$
-m g\left(x^{n}\right) x^{m}-m d\left(x^{n}\right) x^{m}+n d\left(x^{m}\right) x^{n}+n x^{n} g\left(x^{m}\right) \in \mathcal{C}
$$

for all $x \in \mathcal{U}$. Substituting $x+\lambda y$ for $x$ in (2.13) and applying Lemma 2.1 yields

$$
\begin{aligned}
-m g\left(x^{n-1} y+x^{n-2} y x\right. & \left.+\cdots+y x^{n-1}\right) x^{m} \\
& -m d\left(x^{n-1} y+x^{n-2} y x+\cdots+y x^{n-1}\right) x^{m} \\
& -m g\left(x^{n}\right)\left(x^{m-1} y+x^{m-2} y x+\cdots+y x^{m-1}\right) \\
& -m d\left(x^{n}\right)\left(x^{m-1} y+x^{m-2} y x+\cdots+y x^{m-1}\right) \\
& +n d\left(x^{m-1} y+x^{m-2} y x+\cdots+y x^{m-1}\right) x^{n} \\
& +n x^{n} g\left(x^{m-1} y+x^{m-2} y x+\cdots+y x^{m-1}\right) \\
& +n d\left(x^{m}\right)\left(x^{n-1} y+x^{n-2} y x+\cdots+y x^{n-1}\right) \\
& +n\left(x^{n-1} y+x^{n-2} y x+\cdots+y x^{n-1}\right) g\left(x^{m}\right) \in \mathcal{C}
\end{aligned}
$$

for all $x, y \in \mathcal{U}$. Taking $y=e$ into (2.14), we obtain

$$
\begin{aligned}
& -m n g\left(x^{n-1}\right) x^{m}-m n d\left(x^{n-1}\right) x^{m}-m^{2} g\left(x^{n}\right) x^{m-1}-m^{2} d\left(x^{n}\right) x^{m-1} \\
& \quad \operatorname{mnd}\left(x^{m-1}\right) x^{n}+m n x^{n} g\left(x^{m-1}\right)+n^{2} d\left(x^{m}\right) x^{n-1}+n^{2} x^{n-1} g\left(x^{m}\right) \in \mathcal{C}
\end{aligned}
$$


for all $x \in \mathcal{U}$. Continuing the process between (2.13)-(2.15) and applying the fact that $\mathcal{U}$ is torsion free we ultimately get

$$
-(n+m) g(x) x+(n-m) d(x) x-(n-m) x d(x)+(n+m) x g(x) \in \mathcal{C}
$$

for all $x \in \mathcal{U}$. This is

$$
[(n-m) d(x)-(n+m) g(x), x] \in \mathcal{C}
$$

for all $x \in \mathcal{U}$. By [18, Lemma 3] it follows that

$$
(n+m) g(x)=(n-m) d(x)
$$

for all $x \in \mathcal{U}$.

CASE 1. If $m=n$, then $(n+m) g(x)=0$ for all $x \in \mathcal{U}$. Due to torsion free fact of the ring $\mathcal{U}, g(x)=0$. Thus (2.8) becomes as follows

$$
[\mu(e), x]+d(x)=0
$$

for all $x \in \mathcal{U}$. This leads to $\mu(x)=\mu(e) x+d(x)=x \mu(e)$, which means $\mu$ is a right multiplier.

CASE 2. If $m \neq n$, by (2.8), (2.16) and a little computation, we have

$$
\begin{aligned}
& (m+n)[\mu(e), x]+2 m d(x)=0 \\
& (m-n)[\mu(e), x]-2 m g(x)=0
\end{aligned}
$$

for all $x \in \mathcal{U}$. Replacing $x^{m}$ for $x$ in (2.17) and (2.18) leads to

$$
\begin{aligned}
& (m+n)\left[\mu(e), x^{m}\right]+2 m d\left(x^{m}\right)=0 \\
& (m-n)\left[\mu(e), x^{m}\right]-2 m g\left(x^{m}\right)=0
\end{aligned}
$$

for all $x \in \mathcal{U}$. Combining (2.11) with (2.12) we get

$$
2 m \mu(e) x^{m+n}-2 m x^{n} \mu(e) x^{m}+2 m d\left(x^{m}\right) x^{n}+2 m x^{n} g\left(x^{m}\right) \in \mathcal{C} .
$$

By (2.19), (2.20) and (2.21) we obtain

$$
\left(2 m \mu(e) x^{m}-(m+n)\left[\mu(e), x^{m}\right]\right) x^{n}-x^{n}\left(2 m \mu(e) x^{m}-(m-n)\left[\mu(e), x^{m}\right]\right) \in \mathcal{C}
$$

for all $x \in \mathcal{U}$. That is

$$
(n-m)\left[x^{n+m}, \mu(e)\right]-(m+n) x^{m}\left[x^{n-m}, \mu(e)\right] x^{m} \in \mathcal{C}
$$

for all $x \in \mathcal{U}$. Let us set $d_{\mu(e)}(x)=[x, \mu(e)]$. Then (2.22) can be rewritten

$$
(n-m) d_{\mu(e)}\left(x^{m+n}\right)-(m+n) x^{m} d_{\mu(e)}\left(x^{n-m}\right) x^{m} \in \mathcal{C}
$$


for all $x \in \mathcal{U}$. Substituting $x+\lambda y$ for $x$ in (2.23) and applying Lemma 2.1 yields

$$
\begin{aligned}
(n- & m) d_{\mu(e)}\left(x^{n+m-1} y+x^{n+m-2} y x+\cdots+y x^{n+m-1}\right) \\
& -(m+n)\left(x^{m-1} y+x^{m-2} y x \cdots+y x^{m-1}\right) d_{\mu(e)}\left(x^{n-m}\right) x^{m} \\
& -(m+n) x^{m} d_{\mu(e)}\left(x^{n-m}\right)\left(x^{m-1} y+x^{m-2} y x \cdots+y x^{m-1}\right) \\
& -(m+n) x^{m} d_{\mu(e)}\left(x^{n-m-1} y+x^{n-m-2} y x+\cdots+y x^{n-m-1}\right) x^{m} \in \mathcal{C}
\end{aligned}
$$

for all $x \in \mathcal{U}$. Taking $y=e$ into (2.24) leads to

$$
\begin{gathered}
(n-m)(m+n) d_{\mu(e)}\left(x^{n+m-1}\right)-m(m+n) x^{m-1} d_{\mu(e)}\left(x^{n-m}\right) x^{m} \\
-m(m+n) x^{m} d_{\mu(e)}\left(x^{n-m}\right) x^{m-1}-(n-m)(m+n) x^{m} d_{\mu(e)}\left(x^{n-m-1}\right) x^{m} \in \mathcal{C}
\end{gathered}
$$

for all $x \in \mathcal{U}$. Since $\mathcal{U}$ is a $2(m+n)$ !-torsion free ring,

$$
\begin{aligned}
& (n-m) d_{\mu(e)}\left(x^{n+m-1}\right)-m x^{m-1} d_{\mu(e)}\left(x^{n-m}\right) x^{m} \\
& \quad-m x^{m} d_{\mu(e)}\left(x^{n-m}\right) x^{m-1}-(n-m) x^{m} d_{\mu(e)}\left(x^{n-m-1}\right) x^{m} \in \mathcal{C} .
\end{aligned}
$$

for all $x \in \mathcal{U}$. Comparing (2.23) with (2.25), we have by induction

$$
d_{\mu(e)}\left(x^{3}\right)-3 x d_{\mu(e)}(x) x \in \mathcal{C}
$$

for all $x \in \mathcal{U}$. That is

$$
\left[\left[d_{\mu(e)}(x), x\right], x\right] \in \mathcal{C}
$$

for all $x \in \mathcal{U}$. By [15, Theorem 1] it follows that

$$
d_{\mu(e)}(x)=0
$$

for all $x \in \mathcal{U}$. This implies that $\mu(e) \in \mathcal{C}$. In view of (2.17) and (2.18), we have $d=0$ and $g=0$. This shows that $\mu$ and $v$ are right (or left) multipliers.

As a direct corollary of Theorem 2.3 we immediately get

THEOREM 2.4. Let $m, n$ be fixed positive integers, $\mathcal{R}$ be a noncommutative $2(m+n)$ !-torsion free prime ring and $d, g$ be a pair of Jordan derivations on $\mathcal{R}$. If $d\left(x^{m}\right) x^{n}+x^{n} g\left(x^{m}\right) \in \mathcal{Z}$ for all $x \in \mathcal{R}$, then $d=0$ and $g=0$.

Using the orthogonal completeness method [2] we can extend Theorem 2.4 to the case of semiprime rings. 
THeORem 2.5. Let $m, n$ be a fixed positive integer, $\mathcal{R}$ be a noncommutative $2(m+n)$ !-torsion free semiprime ring and $d, g$ be a pair of Jordan derivations on $\mathcal{R}$. If $d\left(x^{m}\right) x^{n}+x^{n} g\left(x^{m}\right) \in \mathcal{Z}$ for all $x \in \mathcal{R}$, then $d$ and $g$ both map $\mathcal{R}$ into $\mathcal{Z}$.

Proof. By Cusack's Theorem [6] we know that $d$ and $g$ are derivations on $\mathcal{R}$. Let $\mathcal{B}$ be the complete Boolean algebra of $\mathcal{E}$. We choose a maximal ideal $\mathcal{M}$ of $\mathcal{B}$. According to [2], $\mathcal{M U}$ is a prime ideal of $\mathcal{U}$, which is invariant under any derivation of $\mathcal{U}$. It is well known that the pair of derivations $d, g$ on $\mathcal{R}$ can be uniquely extended to be a pair of derivations on $\mathcal{U}$. Let $\bar{d}, \bar{g}$ be the canonical pair of derivations on $\overline{\mathcal{U}}=\mathcal{U} / \mathcal{M U}$ induced by $d, g$, respectively. The assumption implies that

$$
\left[d\left(x^{m}\right) x^{n}+x^{n} g\left(x^{m}\right), z\right]=0
$$

for all $x, z \in \mathcal{R}$. It follows from [16, Theorem 2] that $\mathcal{R}$ and $\mathcal{U}$ satisfy the same differential identities. Thus

$$
\left[d\left(x^{m}\right) x^{n}+x^{n} g\left(x^{m}\right), z\right]=0
$$

for all $x, z \in \mathcal{U}$. Furthermore,

$$
\left[\bar{d}\left(\bar{x}^{m}\right) \bar{x}^{n}+\bar{x}^{n} \bar{g}\left(\bar{x}^{m}\right), \bar{z}\right]=0,
$$

for all $\bar{x}, \bar{z} \in \overline{\mathcal{U}}$. By Theorem 2.4 we know that either $\bar{d}(\bar{x})=0$ and $\bar{g}(\bar{x})=0$ or $[\overline{\mathcal{U}}, \overline{\mathcal{U}}]=0$. In any case, we have both

$$
d(\mathcal{U})[\mathcal{U}, \mathcal{U}] \in \mathcal{M U}
$$

and

$$
g(\mathcal{U})[\mathcal{U}, \mathcal{U}] \in \mathcal{M U}
$$

for all $\mathcal{M}$. Note that $\bigcap\{\mathcal{M U} \mid \mathcal{M}$ is any maximal ideal of $\mathcal{B}\}=0$. Hence $d(\mathcal{U})[\mathcal{U}, \mathcal{U}]=0$ and $g(\mathcal{U})[\mathcal{U}, \mathcal{U}]=0$. In particular, $d(\mathcal{R})[\mathcal{R}, \mathcal{R}]=0$ and $g(\mathcal{R})[\mathcal{R}, \mathcal{R}]=0$. These imply that

$$
0=d(\mathcal{R})\left[\mathcal{R}^{2}, \mathcal{R}\right]=d(\mathcal{R}) \mathcal{R}[\mathcal{R}, \mathcal{R}]+d(\mathcal{R})[\mathcal{R}, \mathcal{R}] \mathcal{R}=d(\mathcal{R}) \mathcal{R}[\mathcal{R}, \mathcal{R}]
$$

and

$$
0=g(\mathcal{R})\left[\mathcal{R}^{2}, \mathcal{R}\right]=g(\mathcal{R}) \mathcal{R}[\mathcal{R}, \mathcal{R}]+g(\mathcal{R})[\mathcal{R}, \mathcal{R}] \mathcal{R}=g(\mathcal{R}) \mathcal{R}[\mathcal{R}, \mathcal{R}] .
$$

Therefore $[\mathcal{R}, d(\mathcal{R})] \mathcal{R}[\mathcal{R}, d(\mathcal{R})]=0$ and $[\mathcal{R}, g(\mathcal{R})] \mathcal{R}[\mathcal{R}, g(\mathcal{R})]=0$. By semiprimeness of $\mathcal{R}$ we obtain that $[\mathcal{R}, d(\mathcal{R})]=0$ and $[\mathcal{R}, g(\mathcal{R})]=0$. These show that $d(\mathcal{R}) \in \mathcal{Z}$ and $g(\mathcal{R}) \in \mathcal{Z}$. 
THeOREm 2.6. Let $m, n, p, q$ be fixed non-negative integers, $\mathcal{R}$ be a noncommutative $2(m+n)$ !-torsion free prime ring and $d, g$ be a pair of Jordan derivations on $\mathcal{R}$. If $x^{m} d(x) x^{n}+x^{p} g(x) x^{q} \in \mathcal{Z}$ for all $x \in \mathcal{R}$, then $d=0$ and $g=0$, where $m \neq p$ or $n \neq q$.

Proof. Since $d$ and $g$ are Jordan derivations on $\mathcal{R}, d$ and $g$ also are derivations on $\mathcal{R}$ by Herstein's Theorem [12]. It is well known that $\mathcal{R}$ and $\mathcal{U}$ satisfy the same differential identities [16, Theorem 2]. This implies that

$$
x^{m} d(x) x^{n}+x^{p} g(x) x^{q} \in \mathcal{C}
$$

for all $x \in \mathcal{U}$. Substituting $x+\lambda y$ for $x$ in (2.26) and applying Lemma 2.1 yields

$$
\begin{aligned}
P_{1}(x, y)= & \left(x^{m-1} y+x^{m-2} y x+\cdots+y x^{m-1}\right) d(x) x^{n}+x^{m} d(y) x^{n} \\
& +x^{m} d(x)\left(x^{n-1} y+x^{n-2} y x+\cdots+y x^{n-1}\right) \\
& +\left(x^{p-1} y+x^{p-2} y x+\cdots+y x^{p-1}\right) g(x) x^{q} \\
& +x^{p} g(y) x^{q}+x^{p} g(x)\left(x^{q-1} y+x^{q-2} y x+\cdots+y x^{q-1}\right) \in \mathcal{C}
\end{aligned}
$$

for all $x, y \in \mathcal{U}$, where $P_{i}(x, y)$ denotes the sum of terms involving $i$ factors of $y$. Note that $\mathcal{U}$ has the identity element $e$. Taking $x=e$ into (2.27) we get

$$
d(y)+g(y) \in \mathcal{C}
$$

for all $y \in \mathcal{U}$. By the well known Posner's theorem [18] it follows that

$$
d(y)+g(y)=0
$$

for all $y \in \mathcal{U}$. Using a similar computational way to (2.27), we also have

$$
P_{2}(e, y)=m y d(y)+n d(y) y+p y g(y)+q g(y) y \in \mathcal{C}
$$

for all $y \in \mathcal{U}$. Comparing (2.29) with (2.28) we get

$$
(n-q) d(y) y+(m-p) y d(y) \in \mathcal{C}
$$

for all $y \in \mathcal{U}$. If $n-q=m-p \neq 0$, then $d=0$ by the torsion free fact of $\mathcal{U}$. If $n-q \neq m-p$, linearizing the identity (2.30) yields

$$
(n-q) d(x) y+(n-q) d(y) x+(m-p) x d(y)+(m-p) y d(x) \in \mathcal{C}
$$

for all $x, y \in \mathcal{U}$. Taking $y=e$ into (2.31) leads to

$$
(n-q) d(x)+(m-p) d(x) \in \mathcal{C}
$$


for all $x \in \mathcal{U}$. If $n-q=-m+p$, then (2.30) becomes as follows

$$
[d(y), y] \in \mathcal{C}
$$

for all $y \in \mathcal{U}$. By Posner' theorem we know that $d=0$. If $n-q \neq-m+p$, then (2.32) also make $d=0$. In any case of $m \neq p$ or $n \neq q, d=0$ always holds. So $g=0$ by (2.28).

Similarly, we also adopt the orthogonal completeness method [2] to prove the semiprime version corresponding to Theorem 2.6. Its proof is completely analogous to that of Theorem 2.5 and is not presented here. We only state it as

Theorem 2.7. Let $m, n, p, q$ be fixed non-negative integers, $\mathcal{R}$ be a noncommutative $2(m+n)$ !-torsion free semiprime ring and $d, g$ be a pair of Jordan derivations on $\mathcal{R}$. If $x^{m} d(x) x^{n}+x^{p} g(x) x^{q} \in \mathcal{Z}$ for all $x \in \mathcal{R}$, then $d$ and $g$ both map $\mathcal{R}$ into $\mathcal{Z}$, where $m \neq$ or $n \neq q$.

\section{Pairs of Derivations on Banach Algebras.}

In view of the above algebraic results, we will focus several range inclusion problems involved pair of derivations on Banach algebras in this section. $\mathcal{A}$ always denotes a Banach algebra which is a complex normed algebra and its underlying vector space is a Banach space. The Jacobson radical of $\mathcal{A}$ is the intersection of all primitive ideals of $\mathcal{A}$ and is denoted by $\operatorname{rad}(\mathcal{A})$. The nil radical of $\mathcal{A}$ is the intersection of all prime ideals of $\mathcal{A}$ and is denoted by $\operatorname{nil}(\mathcal{A})$. Let $\mathcal{I}$ be any closed ideal of the Banach algebra $\mathcal{A}$. Then $Q_{\mathcal{I}}$ denotes the canonical quotient map from $\mathcal{A}$ onto $\mathcal{A} / \mathcal{I}$. Moreover, we assume that all mappings on Banach algebra $\mathcal{A}$ are linear mappings in the whole paper.

At present, most of results in this field deal with one derivation, while pair of derivations on Banach algebras was more less considered. On the other hand, many results are proved under the assumption of continuity of derivations. Our several results are proved without assuming the continuity of derivations. Let us begin this section with the following lemma.

LEMma 3.1 [23, Lemma 1.2]. Let d be a derivation on Banach algebra $\mathcal{A}$ and $\mathcal{J}$ be a primitive ideal of $\mathcal{A}$. If there exists a real constant $k>0$ such that $\left\|Q_{\mathcal{J}} d^{n}\right\| \leq k^{n}$ for all $n \in \mathbb{N}$, then $d(\mathcal{J}) \subseteq \mathcal{J}$.

Now we are in position to prove the main result of this section. 
THEOREM 3.2. Let $\mathcal{A}$ be a noncommutative Banach algebra and $d, g$ be a pair of derivations on $\mathcal{A}$. If $d\left(x^{m}\right) x^{n}+x^{n} g\left(x^{m}\right) \in \mathcal{Z}_{\mathcal{A}}$ for all $x \in \mathcal{A}$ and fixed positive integers $m, n$, then $d(\mathcal{A}) \subseteq \operatorname{rad}(\mathcal{A})$ and $g(\mathcal{A}) \subseteq \operatorname{rad}(\mathcal{A})$.

Proof. Let $\mathcal{J}$ be any primitive ideal of $\mathcal{A}$. By Zorn lemma, there exists a minimal prime ideal $\mathcal{P}$ of $\mathcal{A}$ contained in $\mathcal{J}$. Then $d(\mathcal{P}) \subseteq \mathcal{P}$ and $g(\mathcal{P}) \subseteq \mathcal{P}$, by [17, Lemma]. If $\mathcal{P}$ is closed, then $d$ and $g$ induce the derivations on the Banach algebra $\mathcal{A} / \mathcal{P}$ as follows

$$
\tilde{d}(\tilde{x})=d(x)+\mathcal{P}, \quad \tilde{g}(\tilde{x})=g(x)+\mathcal{P}
$$

for all $\tilde{x} \in \mathcal{A} / \mathcal{P}$ and $x \in \mathcal{A}$. When $\mathcal{A} / \mathcal{P}$ is commutative, both $\tilde{d}(\mathcal{A} / \mathcal{P})$ and $\tilde{g}(\mathcal{A} / \mathcal{P})$ are contained in the Jacobson radical of $\mathcal{A} / \mathcal{P}$ by [22, Theorem 4.4]. When $\mathcal{A} / \mathcal{P}$ is noncommutative, the assumption yields

$$
\left[\tilde{d}\left(\tilde{x}^{m}\right) \tilde{x}^{n}+\tilde{x}^{n} \tilde{g}\left(\tilde{x}^{m}\right), \tilde{z}\right]=\tilde{0}
$$

for all $\tilde{x}, \tilde{z} \in \mathcal{A} / \mathcal{P}$ and $x, z \in \mathcal{A}$. By the primeness of $\mathcal{A} / \mathcal{P}$ and Theorem 2.4, it follows that $\tilde{d}=\tilde{0}$ and $\tilde{g}=\tilde{0}$ on $\mathcal{A} / \mathcal{P}$. In any case, we get both $d(\mathcal{A}) \subseteq \mathcal{J}$ and $g(\mathcal{A}) \subseteq \mathcal{J}$. If $\mathcal{P}$ is not closed, then $\mathcal{S}(d) \subseteq \mathcal{P}$ by [6, Lemma 2.3], where $\mathcal{S}(d)$ is the separating space of linear operator $d$. By [20, Lemma 1.3], we have $\left.\mathcal{S}\left(Q_{\hat{\mathcal{P}}} d\right)=Q_{\hat{\mathcal{P}}} \widehat{(\mathcal{S}(d)}\right)=0$, whence $Q_{\hat{\mathcal{P}}} d$ is continuous on $\mathcal{A}$. This implies that $Q_{\hat{\mathcal{P}}} d(\hat{\mathcal{P}})=0$ on $\mathcal{A} / \mathcal{P}$ and hence $d(\hat{\mathcal{P}}) \subseteq \hat{\mathcal{P}}$. Thus $d$ induces a derivation on the Banach algebra $\mathcal{A} / \hat{\mathcal{P}}$ as follows

$$
\tilde{d}(\tilde{x})=d(x)+\hat{\mathcal{P}}
$$

for all $\tilde{x} \in \mathcal{A} / \hat{\mathcal{P}}$ and $x \in \mathcal{A}$. Thus we can define the following map

$$
\xi \tilde{d}^{n} Q_{\hat{\mathcal{P}}}: \mathcal{A} \longrightarrow \mathcal{A} / \hat{\mathcal{P}} \longrightarrow \mathcal{A} / \hat{\mathcal{P}} \longrightarrow \mathcal{A} / \mathcal{J}
$$

through $\xi \tilde{d}^{n} Q_{\hat{\mathcal{P}}}(x)=Q_{\mathcal{J}} d^{n}(x)$ for all $x \in \mathcal{A}$ and $n \in \mathbb{N}$, where $\xi$ is the canonical inclusion map from $\mathcal{A} / \hat{\mathcal{P}}$ onto $\mathcal{A} / \mathcal{J}$ and $\xi$ indeed exists since $\hat{\mathcal{P}} \subseteq \mathcal{J}$. By [20, Lemma 1.4], we claim that $\tilde{d}$ is continuous on $\mathcal{A} / \hat{\mathcal{P}}$ and hence that $\left\|Q_{\mathcal{J}} d^{n}\right\| \leq\|\tilde{d}\|^{n}$ for all $n \in \mathbb{N}$. By Lemma 3.1, we obtain $d(\mathcal{J}) \subseteq \mathcal{J}$. Using the same argument with $g$, we also obtain $g(\mathcal{J}) \subseteq \mathcal{J}$. Then the derivations $d$ and $g$ induce the derivations on the Banach algebra $\mathcal{A} / \mathcal{J}$ as follows

$$
\tilde{d}(\tilde{x})=d(x)+\mathcal{J}, \quad \tilde{g}(\tilde{x})=g(x)+\mathcal{J}
$$

for all $\tilde{x} \in \mathcal{A} / \mathcal{J}$ and $x \in \mathcal{A}$. The remainder follows by a similar argument to the case when $\mathcal{P}$ is closed since the primitive algebra $\mathcal{A} / \mathcal{J}$ is prime. Therefore we conclude that $d(\mathcal{A}) \subseteq \mathcal{J}$ and $g(\mathcal{A}) \subseteq \mathcal{J}$. It follows that 
$d(\mathcal{A}) \subseteq \mathcal{J}$ and $g(\mathcal{A}) \subseteq \mathcal{J}$ for every primitive ideal $\mathcal{J}$, that is, $d(\mathcal{A}) \subseteq \operatorname{rad}(\mathcal{A})$ and $g(\mathcal{A}) \subseteq \operatorname{rad}(\mathcal{A})$. This completes the proof of the theorem.

As a consequence of the above theorem, we get

COROLlary 3.3. Let $\mathcal{A}$ be a noncommutative semisimple Banach algebra and $d, g$ be a pair of derivations on $\mathcal{A}$. If $d\left(x^{m}\right) x^{n}+$ $+x^{n} g\left(x^{m}\right) \in \mathcal{Z}_{\mathcal{A}}$ for all $x \in \mathcal{A}$ and fixed positive integers $m, n$, then $d=0$ and $g=0$.

REMARK 3.4. Simulating the preceding proof ways which just appear in this section, some similar results can be obtained. In fact, Theorem 3.2 and Corollary 3.3 still hold if the condition $d\left(x^{m}\right) x^{n}+x^{n} g\left(x^{m}\right) \in \mathcal{Z}_{\mathcal{A}}$ for all $x \in \mathcal{A}$ is replaced by the condition $d\left(x^{m}\right) x^{n}+x^{n} g\left(x^{m}\right) \in \operatorname{nil}(\mathcal{A})$ for all $x \in \mathcal{A}$. Here the assumption of continuity of derivations is not necessary. We only state the final results without detailed proof.

THEOREM 3.5. Let $\mathcal{A}$ be a noncommutative Banach algebra and $d, g$ be a pair of derivations on $\mathcal{A}$. If $d\left(x^{m}\right) x^{n}+x^{n} g\left(x^{m}\right) \in \operatorname{nil}(\mathcal{A})$ for all $x \in \mathcal{A}$ and fixed positive integers $m, n$, then $d(\mathcal{A}) \subseteq \operatorname{rad}(\mathcal{A})$ and $g(\mathcal{A}) \subseteq \operatorname{rad}(\mathcal{A})$.

COROLlary 3.6. Let $\mathcal{A}$ be a noncommutative semisimple Banach algebra and $d, g$ be a pair of derivations on $\mathcal{A}$. If $d\left(x^{m}\right) x^{n}+$ $+x^{n} g\left(x^{m}\right) \in \operatorname{nil}(\mathcal{A})$ for all $x \in \mathcal{A}$ and fixed positive integers $m, n$, then $d=0$ and $g=0$.

Let us consider the case of a pair of Jordan derivations on Banach algebras.

THEOREM 3.7. Let $\mathcal{A}$ be a noncommutative Banach algebra and $d, g$ be a pair of continuous Jordan derivations on $\mathcal{A}$. If $d\left(x^{m}\right) x^{n}+x^{n} g\left(x^{m}\right) \in$ $\in \operatorname{rad}(\mathcal{A})$ for all $x \in \mathcal{A}$ and fixed positive integers $m, n$, then $d(\mathcal{A}) \subseteq \operatorname{rad}(\mathcal{A})$ and $g(\mathcal{A}) \subseteq \operatorname{rad}(\mathcal{A})$.

Proof. Let $\mathcal{P}$ be any primitive ideal of $\mathcal{A}$. Since $d$ and $g$ are continuous, $d(\mathcal{P}) \subseteq \mathcal{P}$ and $g(\mathcal{P}) \subseteq \mathcal{P}$ by [19, Lemma 3.2]. Then $d$ and $g$ can be induced to the Jordan derivations on the Banach algebra $\mathcal{A} / \mathcal{P}$ as follows

$$
\tilde{d}(\tilde{x})=d(x)+\mathcal{P}, \quad \tilde{g}(\tilde{x})=g(x)+\mathcal{P}
$$

for all $\tilde{x} \in \mathcal{A} / \mathcal{P}$ and $x \in \mathcal{A}$. Since $\mathcal{P}$ is a primitive ideal, the quotient algebra 
$\mathcal{A} / \mathcal{P}$ is prime and semisimple. Thus both $\tilde{d}$ and $\tilde{g}$ are derivations by $[12$, Theorem 3.1]. It has been proved that every derivation on a semisimple Banach algebra is continuous [13, Remark 4.3]. Combing this result with the well known Singer-Wermer theorem, we conclude that there are no nonzero derivations on a commutative semisimple Banach algebra. Hence we have $\tilde{d}=0$ and $\tilde{g}=0$ when $\mathcal{A} / \mathcal{P}$ is commutative. It remains to show that $\tilde{d}=0$ and $\tilde{g}=0$ in the case when $\mathcal{A} / \mathcal{P}$ is noncommutative. The assumption of the theorem yields

$$
\tilde{d}\left(\tilde{x}^{m}\right) \tilde{x}^{n}+\tilde{x}^{n} g\left(\tilde{x}^{m}\right)=\tilde{0}
$$

for all $\tilde{x} \in \mathcal{A} / \mathcal{P}$ and $x \in \mathcal{A}$. It follows from Theorem 2.4 that $\tilde{d}=0$ and $\tilde{g}=0$. In any case both $\tilde{d}=0$ and $\tilde{g}=0$. This implies that $d(\mathcal{A}) \subseteq \mathcal{P}$ and $g(\mathcal{A}) \subseteq \mathcal{P}$. Since $\mathcal{P}$ is arbitrary, $d(\mathcal{A}) \subseteq \operatorname{rad}(\mathcal{A})$ and $g(\mathcal{A}) \subseteq \operatorname{rad}(\mathcal{A})$. This completes the proof of the theorem.

The following corollary is a special case of Theorem 3.7.

Corollary 3.8. Let $\mathcal{A}$ be a noncommutative semisimple Banach algebra and $d, g$ be a pair of Jordan derivations on $\mathcal{A}$. If

$$
d\left(x^{m}\right) x^{n}+x^{n} g\left(x^{m}\right) \in \operatorname{rad}(\mathcal{A})
$$

for all $x \in \mathcal{A}$ and fixed positive integers $m, n$, then $d=0$ and $g=0$.

\section{REFERENCES}

[1] E. AlBAS - N. ARGAC, Generalized derivations of prime rings, Algebra Colloq., 11 (2004), pp. 399-410.

[2] K. I. BEIDAR, Rings of quotients of semiprime rings, Vestnik Moskov. Univ. Ser I Mat. Meh. (Engl. Transl. Moscow Univ. Math. Bull.), 33 (1978), pp. 3642.

[3] M. BREŠAR, Jordan mappings of semiprime rings, J. Algebra, 127 (1989), pp. $218-228$.

[4] D. S. Bridges - J. Bergen, On the derivation of $x^{n}$ in a ring, Proc. Amer. Math. Soc., 90 (1984), pp. 25-29.

[5] L. O. Chung - J. LuH, Semiprime rings with nilpotent derivations, Canad. Math. Bull., 24 (1981), pp. 415-421.

[6] J. CuSACK, Jordan derivations on rings, Proc. Amer. Math. Soc., 53 (1975), pp. $321-324$.

[7] V. DE FILIPPIS, An Engel condition with generalized derivations on multilinear polynomials, Israel J. Math., 162 (2007), pp. 93-108.

[8] V. DE FiLIPPIS, Posner's second theorem and an annihilator condition with generalized derivations, Turkish J. Math., 32 (2008), pp. 197-211. 
[9] V. DE FILIPPIS, Generalized derivations in prime rings and noncommutative Banach algebras, Bull. Korean Math. Soc., 45 (2008), pp. 621-629.

[10] V. DE FILIPPIS - H. G. INCEBOZ, Generalized derivations with power central values on multilinear polynomials on right ideals, Rend. Sem. Mat. Univ. Padova, 120 (2008), pp. 59-71.

[11] V. De Filippis - M. S. TAmmam El-Sayiad, A note on Posner's theorem with generalized derivations on Lie ideals, Rend. Sem. Mat. Univ. Padova, 122 (2009), pp. 55-64.

[12] I. N. Herstein, Jordan derivations of prime rings, Proc. Amer. Math. Soc., 8 (1957), pp. 1104-1110.

[13] B. E. Johnson - A. M. Sinclain, Continuity of derivations and a problem of Kaplansky, Amer J. Math., 90 (1968), pp. 1067-1073.

[14] K. -W. Jun - H. - M. KIM, Derivations on prime rings and Banach algebras, Bull. Korean Math. Soc., 38 (2001), pp. 709-718.

[15] C. Lanski, An Engel condition with derivation, Proc. Amer. Math. Soc., 118 (1993), pp. 731-734.

[16] T. K. LEE, Semiprime rings with differential identities, Bull. Inst. Math. Acad. Sinica, 20 (1992), pp. 27-38.

[17] M. Mathieu - V. Runde, Derivations mapping into the radical, II, Bull. London Math. Soc., 24 (1992), pp. 485-487.

[18] E. Posner, Derivations in prime rings, Proc. Amer. Math. Soc., 8 (1957), pp. 1093-1100.

[19] A. M. Sinclair, Jordan homomorphisms and derivations on semisimple Banach algebras, Proc. Amer. Math. Soc., 24 (1970), pp. 209-214.

[20] A. M. SinclaIR, Automatic Continuity of Linear Operators, London Mathematical Society Lecture Note Series, V. 21, Cambridge University Press (Cambridge, 1976).

[21] I. M. Singer - J. Wermer, Derivations on commutative normed algebras, Math. Ann., 129 (1955), pp. 260-264.

[22] M. P. Thomas, The image of a derivation is contained in the radical, Ann. Math., 128 (1988), pp. 435-460.

[23] M. P. Thomas, Primitive ideals and derivations on noncommutative Banach algebras, Pacific. J. Math., 159 (1993), pp. 139-152.

[24] J. Vukman, Identities with derivations on rings and Banach algebras, Glasnik Matematicki, 40 (2005), pp. 189-199.

[25] J. Vukman - I. Kosi-Ulbu, A note on derivations in semiprime rings, Int. J. Math. Math. Sci., 20 (2005), pp. 3347-3350.

[26] F. WEI, Generalized derivations with nilpotent values on semiprime rings, Acta Math. Sinica, 20 (2004), pp. 453-462.

[27] F. WEI - Z. -K. XIAO, Generalized Jordan (triple-)derivations and pairs of Jordan derivations on semiprime rings, Demonstratio Math., in press.

[28] F. WEI - Z. -K. XIAO, Pair of (Generalized-) Derivations on Rings and Banach Algebras, Bull. Korean Math. Soc., 46 (2009), pp. 857-866.

Manoscritto pervenuto in redazione il 2 dicembre 2008. 